TRANSACTIONS OF THE

AMERICAN MATHEMATICAL SOCIETY

Volume 352 , Number 12, Pages 5767-5780

S 0002-9947(00)02690-8

Article electronically published on August 21, 2000

\title{
THE RANGE OF TRACES ON QUANTUM HEISENBERG MANIFOLDS
}

\author{
BEATRIZ ABADIE
}

\begin{abstract}
We embed the quantum Heisenberg manifold $D_{\mu \nu}^{c}$ in a crossed product $\mathrm{C}^{*}$-algebra. This enables us to show that all tracial states on $D_{\mu \nu}^{c}$ induce the same homomorphism on $K_{0}\left(D_{\mu \nu}^{c}\right)$, whose range is the group $\mathbf{Z}+$ $2 \mu \mathbf{Z}+2 \nu \mathbf{Z}$.
\end{abstract}

\section{INTRODUCTION}

For a positive integer $c$, let $M_{c}$ denote the Heisenberg manifold consisting of the quotient $G / H_{c}$, where $G$ is the Heisenberg group,

$$
G=\left\{\left(\begin{array}{ccc}
1 & y & z \\
0 & 1 & x \\
0 & 0 & 1
\end{array}\right): x, y, z \in R\right\},
$$

and $H_{c}$ is the subgroup of $G$ obtained when $x, y$, and $c z$ are integers.

In [RF3] Rieffel constructed a quantization deformation $\left\{D_{\mu \nu}^{c, \hbar}\right\}_{\hbar \in R}$ of $M_{c}$ in the direction of a given Poisson bracket $\Lambda_{\mu \nu}$ determined by two real parameters $\mu$ and $\nu$. We drop from now on the Planck constant $\hbar$ from our notation, because the algebras $D_{\mu \nu}^{c, \hbar}$ and $D_{\hbar \mu, \hbar \nu}^{c, 1}$ are isomorphic and we will denote either one by $D_{\hbar \mu, \hbar \nu}^{c}$. Also, since $D_{\mu \nu}^{c} \cong D_{\mu+n, \nu+m}^{c}$ for any integers $n$ and $m$ ([AB1] ), we view the parameters $\mu$ and $\nu$ as running in the circle $\mathbf{T}$.

We discussed the K-theory of the quantum Heisenberg manifolds in AB2 and found that $K_{0}\left(D_{\mu \nu}^{c}\right)=\mathbf{Z}^{3} \oplus \mathbf{Z}_{c}$ and $K_{1}\left(D_{\mu \nu}^{c}\right)=\mathbf{Z}^{3}$, which shows that two algebras corresponding to deformations of different Heisenberg manifolds are not isomorphic. In AB1] we constructed finitely generated projective modules over the algebra $D_{\mu \nu}^{c}$ with traces $2 \mu$ and $2 \nu$ respectively, where the trace considered was that defined in RF3. This suggests employing the range of traces on $K_{0}\left(D_{\mu \nu}^{c}\right)$ as an invariant to discuss isomorphism and strong-Morita equivalence types of the family $\left\{D_{\mu \nu}^{c}\right\}$, as was done for non-commutative tori ([PV], [RF1]) and Heisenberg $\mathrm{C}^{*}$-algebras ([PA2, [PA1]).

This work is organized as follows. In Section 2 we embed the algebra $D_{\mu \nu}^{c}$ in a crossed product. This is done in a more general context, by viewing the quantum Heisenberg manifolds as generalized fixed-point algebras, as in [RF3]. In Section 3

Received by the editors December 2, 1996.

2000 Mathematics Subject Classification. Primary 46L80; Secondary 46L55.

Partially supported by Conicyt (Proyecto 2002), Uruguay. Part of the material in this work was contained in the author's Ph.D. dissertation submitted to the University of California at Berkeley in May 1992. 
we show that all traces on $D_{\mu \nu}^{c}$ give rise to the same homomorphism on $K_{0}\left(D_{\mu \nu}^{c}\right)$, whose range is the group $\mathbf{Z}+2 \mu \mathbf{Z}+2 \nu \mathbf{Z}$.

\section{The Embedding}

The purpose of this section is to embed each quantum Heisenberg manifold in a crossed product algebra $A \rtimes \mathbf{Z}, A$ being a $\mathrm{C}^{*}$-subalgebra of $L^{\infty}\left(\mathbf{T}^{2}\right)$. Our construction carries over into a somewhat more general context, which we next describe.

We first recall some facts established in $\mathrm{AB} 2$. Let $\lambda$ and $\sigma$ be two commuting automorphisms of a $\mathrm{C}^{*}$-algebra $B$. Let $u: \mathbf{Z} \times \mathbf{Z} \longrightarrow U \mathbf{Z M}(B)$ be a $\lambda$-cocycle in the first variable and a $\sigma$-cocycle in the second one, and define the action $\gamma^{\sigma, u}$ of $\mathbf{Z}$ on $B \rtimes_{\lambda} \mathbf{Z}$ by $\left(\gamma_{k}^{\sigma, u} \Phi\right)(p)=u(p, k) \sigma_{k}[\Phi(p)]$. When the $\mathrm{C}^{*}$-algebra $B=C_{0}(M)$ is commutative and the actions $\lambda$ and $\sigma$ are free and proper, then $\gamma^{\sigma, u}$ is proper and the corresponding generalized fixed-point algebra $D^{\sigma, u}$, in the sense of Rieffel $([\mathrm{RF} 4])$, is the closure in the multiplier algebra $\mathcal{M}\left(C_{0}(M) \rtimes_{\lambda} \mathbf{Z}\right)$ of the ${ }^{*}$-subalgebra $C^{\sigma, u}$ consisting of functions $\Phi \in C_{c}(\beta M \times \mathbf{Z})$ such that the projection of $\operatorname{supp}_{M}(\Phi)$ on $M / \sigma$ is precompact and $\gamma_{k}^{\sigma, u} \Phi=\Phi$ for all $k \in \mathbf{Z}$, where $\gamma^{\sigma, u}$ has been extended to the multiplier algebra, and $\beta M$ denotes the Stone-Čech compactification of $M$.

When the space $M$ is taken to be $\mathbf{R} \times \mathbf{T}$, and $\sigma(x, y)=(x-1, y), \lambda(x, y)=$ $(x+2 \mu, y+2 \nu)$, and $u(p, k)=\exp (2 \pi i c k p(y-p \nu))$ for $(x, y) \in \mathbf{R} \times \mathbf{T}, k, p \in \mathbf{Z}$, then $D^{\sigma, u}$ is the quantum Heisenberg manifold denoted in [RF3] by $D_{\mu \nu}^{c}$, and we denote by $C_{\mu \nu}^{c}$ the dense ${ }^{*}$-subalgebra corresponding to $C^{\sigma, u}$.

In the general case, if $F$ is a fundamental domain in $M$ for the action $\sigma$ (i.e. the canonical projection $\Pi: F \longrightarrow M / \sigma$ is a bijection), then any $\Phi$ in the dense subalgebra $C^{\sigma, u}$ is determined by the values $\Phi(m, p)$, for $m$ running in $F$ and $p \in \mathbf{Z}$. This suggests the idea of untwisting those functions so that they can be viewed as functions on the quotient space $M / \sigma$. A natural way of doing that is by multiplying them by a function $H$ on $M$ satisfying the opposite condition $\gamma^{\sigma, u^{*}} H=H$. Also, in order to get things to work from an algebraic point of view, it is necessary for $H$ to satisfy

$$
\bar{H}_{-p}\left(\lambda_{-p} m\right)=H_{p}(m) \quad \text { and } \quad H_{p+q}(m)=H_{p}(m) H_{q}\left(\lambda_{-p} m\right) .
$$

However, there might not be such a continuous function on $M$. This is the case for quantum Heisenberg manifolds. If a continuous map $H$ as above existed, then multiplication by the function $\gamma \in C(\mathbf{R} \times \mathbf{T})$ defined by $\gamma(x, y)=H_{1}(x, y+\nu)$ would be a $C\left(\mathbf{T}^{2}\right)$-module isomorphism between $C\left(\mathbf{T}^{2}\right)$ and $X=\{\Phi \in C(\mathbf{R} \times \mathbf{T})$ : $\Phi(x+1, y)=\exp (2 \pi i c y) \Phi(x, y)\}$, in contradiction with [RF2, 3.9].

This is the reason why we are forced to get out of $C_{0}(M / \sigma)$ and consider a bigger subalgebra of $L^{\infty}(M / \sigma)$, as was done in [CU, 2.5] for the case of non-commutative tori.

Measurability considerations will impose some restrictions on the fundamental domain $F$. We next summarize the assumptions we will be making.

Assumptions and notation. In what follows, for a $\mathrm{C}^{*}$-algebra $A$ we denote by $\mathcal{M}(A)$ its multiplier algebra, and by $\mathcal{U}(A)$ the group of unitary elements in $A$.

Throughout this section $\lambda$ and $\sigma$ denote free and proper commuting actions of $\mathbf{Z}$ on a locally compact Hausdorff space $M$, and $u: \mathbf{Z} \times \mathbf{Z} \longrightarrow \mathcal{U} M\left(C_{0}(M)\right)$ denotes a map satisfying the cocycle conditions:

$$
u(p+q, k)=u(p, k) \lambda_{p}[u(q, k)] \quad \text { and } \quad u(p, k+l)=u(p, k) \sigma_{k}[u(p, l)],
$$


for any $k, l, p, q \in \mathbf{Z}$, where $\sigma$ has been extended to the multiplier algebra. We also assume the existence of a Borel measurable fundamental domain $F$ for $\sigma$ in $M$ such that the canonical projection $\Pi: F \longrightarrow M / \sigma$ has a Borel measurable inverse map. Thus a function $f$ on $M / \sigma$ is Borel measurable if and only if $f=\tilde{f} \circ \Pi$, for some Borel measurable function $\tilde{f}$ on $M$.

The generalized fixed-point algebra of $C_{0}(M) \rtimes_{\lambda} \mathbf{Z}$ under the action $\gamma^{\sigma, u}$ of $\mathbf{Z}$ defined by $\left(\gamma_{k}^{\sigma, u} \Phi\right)(m, p)=u(p, k) \Phi\left(\sigma_{-k} m, p\right)$, for $\Phi \in C_{c}(M \times \mathbf{Z})$ will be denoted by $D^{\sigma, u}$. We denote by $C^{\sigma, u}$ the dense *-subalgebra of $D^{\sigma, u}$ consisting of functions $\Phi \in C_{c}(\beta M \times \mathbf{Z})$ such that the projection of $\operatorname{supp}_{M}(\Phi)$ on $M / \sigma$ is precompact and that $\gamma_{k}^{\sigma, u} \Phi=\Phi$, for all $k \in \mathbf{Z}$.

Lemma 2.1. Let $H: \mathbf{Z} \rightarrow \mathcal{U} L^{\infty}(M)$ be defined by: $H_{1}(m)=u^{*}(1, k)(m)$, for $m \in \sigma_{k} F$, and

$$
H_{p}(m)= \begin{cases}\prod_{q=0}^{p-1}\left(\lambda_{q} H_{1}\right)(m) & \text { if } p>0 \\ 1 & \text { if } p=0 \\ \prod_{q=p}^{-1} \overline{\left(\lambda_{q} H_{1}\right)}(m) & \text { if } p<0\end{cases}
$$

Then:

i) $H$ is a $\lambda$-cocycle (i.e. $H_{p+q}(m)=H_{p}(m) H_{q}\left(\lambda_{-p} m\right)$ for all $m \in M, p, q \in \mathbf{Z}$ ).

ii) $\bar{H}_{-p}\left(\lambda_{-p} m\right)=H_{p}(m)$, for all $m \in M$ and $p \in \mathbf{Z}$.

iii) $H_{p}\left(\sigma_{-k} m\right)=\left[u(p, k) H_{p}\right](m)$, for all $m \in M$ and $k, p \in \mathbf{Z}$.

Proof. i) For $q=1$ and $p>0$, we have

$$
H_{p+1}(m)=\prod_{q=0}^{p}\left(\lambda_{q} H_{1}\right)(m)=H_{p}(m)\left(\lambda_{-p} H_{1}\right)(m)=H_{p}(m) H_{1}\left(\lambda_{-p} m\right) .
$$

An analogous computation shows that the equality holds for $p \leq 0$, and, once ii) is proven, the result follows by induction on $q$.

It suffices to prove ii) for $p>0$, and in that case we have

$$
\bar{H}_{-p}\left(\lambda_{-p} m\right)=\prod_{q=-p}^{q=-1}\left(\lambda_{p+q} H_{1}\right)(m)=\prod_{q=0}^{q=p-1}\left(\lambda_{q} H_{1}\right)(m)=H_{p}(m) .
$$

Finally, for $p>0$, we have

$$
\begin{aligned}
H_{p}\left(\sigma_{-k} m\right) & =\prod_{q=0}^{p-1}\left(\lambda_{q} H_{1}\right)\left(\sigma_{-k} m\right) \\
& =\prod_{q=0}^{p-1}\left[\lambda_{q}(u(1, k))\left(\lambda_{q} H_{1}\right)\right](m) \\
& =u(p, k) H_{p}(m) .
\end{aligned}
$$

This ends the proof in view of ii).

Notation 2.2. Let $H$ be as in Lemma 2.1. For $p \in \mathbf{Z}$ and $\Phi \in C^{\sigma, u}$ let $f_{\Phi, p} \in$ $L^{\infty}(M / \sigma)$ be defined by $f_{\Phi, p}(\dot{m})=H_{p}(m) \Phi(m, p)$, where $\dot{m}$ denotes the projection of $m$ onto $M / \sigma$. 
Theorem 2.3. Let $H$ be as in Lemma[2.1. Then the generalized fixed-point algebra $D^{\sigma, u}$ can be embedded in the crossed product $A \rtimes_{\lambda} \mathbf{Z}$, where $A$ is any $\lambda$-invariant $C^{*}$-subalgebra of $L^{\infty}(M / \sigma)$ containing $\left\{f_{\Phi, p}: \Phi \in C^{\sigma, u}, p \in \mathbf{Z}\right\}$.

Proof. Let $J: D^{\sigma, u} \longrightarrow A \rtimes_{\lambda} \mathbf{Z}$ be defined, at the level of functions $\Phi \in C^{\sigma, u}$, by $(J \Phi)(\dot{m}, p)=f_{\Phi, p}(\dot{m})$. Then, by properties i) and ii) in Lemma $2.1 J$ is a ${ }^{*}$-algebra homomorphism:

$$
\begin{aligned}
\left(J \Phi^{*}\right)(\dot{m}, p) & =H_{p}(m) \bar{\Phi}\left(\lambda_{-p} m,-p\right) \\
& =\bar{H}_{-p}\left(\lambda_{-p} m\right) \bar{\Phi}\left(\lambda_{-p} m,-p\right) \\
& =(J \Phi)^{*}(\dot{m}, p)
\end{aligned}
$$

and

$$
\begin{aligned}
J(\Phi * \Psi)(\dot{m}, p) & =\sum_{q \in \mathbf{Z}} H_{q}(m) H_{p-q}\left(\lambda_{-q} m\right) \Phi(m, q) \Psi\left(\lambda_{-q} m, p-q\right) \\
& =H_{p}(m)(\Phi * \Psi)(m, p) \\
& =[J(\Phi * \Psi)](\dot{m}, p) .
\end{aligned}
$$

Let $\mu_{0}$ be a Borel measure of full support on $F$ and, for $\sigma_{k}: F \rightarrow \sigma_{k} F$ and $\Pi$ : $F \rightarrow M / \sigma$, set $\mu_{k}=\left(\sigma_{k}\right)_{*}\left(\mu_{0}\right)$ and $\tilde{\mu}=\Pi_{*}\left(\mu_{0}\right)$. Then $\tilde{\mu}$ and $\mu_{k}$ have full support on $M / \sigma$ and $\sigma_{k} F$ respectively, for all $k \in \mathbf{Z}$. In what follows we will also denote by $\mu_{k}$ the Borel measure on $M$ obtained by setting $\mu_{k}(X)=\mu_{k}\left(X \cap \sigma_{k} F\right)$, for a Borel subset $X$ of $M$. Now let $\tilde{\Theta}$ and $\Theta^{k}$, for $k \in \mathbf{Z}$, denote the representations of $A \rtimes_{\lambda} \mathbf{Z}$ and $D^{\sigma, u}$ on $L^{2}(M / \sigma \times \mathbf{Z}, \tilde{\mu} \times \nu)$ and $L^{2}\left(M \times \mathbf{Z}, \mu_{k} \times \nu\right)$ ( $\nu$ being counting measure on $\mathbf{Z})$, respectively, defined by

$$
\left(\tilde{\Theta}_{\Psi} \xi\right)(\dot{m}, p)=\sum_{q \in \mathbf{Z}} \Psi\left(\lambda_{p} \dot{m}, q\right) \xi(\dot{m}, p-q)
$$

and

$$
\left(\Theta_{\Phi}^{k} \eta\right)(m, p)=\sum_{q \in \mathbf{Z}} \Phi\left(\lambda_{p} m, q\right) \eta(m, p-q),
$$

where $\Phi \in C^{\sigma, u}, \Psi \in C_{c}(M / \sigma \times \mathbf{Z}), \xi \in L^{2}(M / \sigma \times \mathbf{Z}, \tilde{\mu} \times \nu)$, and moreover $\eta \in L^{2}\left(M \times \mathbf{Z}, \mu_{k} \times \nu\right)$. Let $U: L^{2}(M / \sigma \times \mathbf{Z}, \tilde{\mu} \times \nu) \rightarrow L^{2}\left(M \times \mathbf{Z}, \mu_{k} \times \nu\right)$ be the unitary operator defined by $(U \xi)(m, p)=\bar{H}_{p}\left(\lambda_{p} m\right) \xi(\dot{m}, p)$. Then, if $m \in \sigma_{k} F$, we have

$$
\begin{aligned}
\left|\tilde{\Theta}_{J \Phi} \xi(\dot{m}, p)\right| & =\left|\sum_{q \in \mathbf{Z}}(J \Phi)\left(\lambda_{p} \dot{m}, q\right) \xi(\dot{m}, p-q)\right| \\
& =\left|\sum_{q \in \mathbf{Z}} H_{q}\left(\lambda_{p} m\right) \Phi\left(\lambda_{p} m, q\right)(U \xi)(m, p-q) H_{p-q}\left(\lambda_{p-q} m\right)\right| \\
& =\left|\sum_{q \in \mathbf{Z}} H_{p}\left(\lambda_{p} m\right) \Phi\left(\lambda_{p} m, q\right)(U \xi)(m, p-q)\right| \\
& =\left|\Theta_{\Phi}^{k}(U \xi)(m, p)\right|,
\end{aligned}
$$

and it follows that $\left\|\tilde{\Theta}_{J \Phi} \xi\right\|=\left\|\Theta_{\Phi}^{k}(U \xi)\right\|$.

Now, the representation $\tilde{\Theta}$ is faithful ([D, 7.7.5, 7.7.7]); therefore, for $\Phi \in C^{\sigma, u}$,

$$
\|J \Phi\|=\left\|\tilde{\Theta}_{J \Phi}\right\|=\left\|\Theta_{\Phi}^{k}\right\| \leq\|\Phi\|
$$

so $J$ can be extended to a continuous map on $D^{\sigma, u}$. 
We next show that, for $\Phi \in C^{\sigma, u}$, we have $\|\Phi\|=\sup _{k}\left\|\Theta_{\Phi}^{k}\right\|=\|J \Phi\|$, which takes care of the injectivity of $J$.

First notice that the representation $\bigoplus_{k} \Theta^{k}$ is unitarily equivalent to the representation $\Theta$ of $D^{\sigma, u}$ on $L^{2}(M \times \mathbf{Z}, \mu \times \nu)$ defined by the same formula as $\Theta^{k}$, where, for a Borel subset $X$ of $M$, we set $\mu(X)=\sum_{k} \mu_{k}\left(X \cap \sigma_{k} F\right)$.

Thus it suffices to prove that $\Theta$ is faithful. In order to do that, we show (PD) 7.7.5, 7.7.7]) that $\mu$ has full support on $M$ : Let $O \subset M$ be an open set such that $\mu(O)=0$. Then, for all $k \in \mathbf{Z}$, we have that $O \cap \sigma_{k} F$ is an open subset of $\sigma_{k} F$ and $\mu_{k}\left(O \cap \sigma_{k} F\right)=0$. Since $\mu_{k}$ has full support on $\sigma_{k} F$, it follows that $A=\bigcup A \cap \sigma_{k} F=\emptyset$, which ends the proof.

From now on we will be dealing with the case of quantum Heisenberg manifolds. We specialize Theorem 2.3 to that case.

Corollary 2.4. Let $\lambda$ be the action of $\mathbf{Z}$ on $\mathbf{T}^{2}$ defined by

$$
\lambda_{k}(x, y)=(x+2 k \mu, y+2 k \nu)
$$

and let $A$ denote the smallest $\lambda$-invariant $C^{*}$-subalgebra of $L^{\infty}\left(\mathbf{T}^{2}\right)$ containing $C\left(\mathbf{T}^{2}\right)$ and the characteristic functions of the sets $[2 k \mu, 2(k+1) \mu] \times \mathbf{T}$, for all $k \in \mathbf{Z}$. Then the quantum Heisenberg manifold $D_{\mu \nu}^{c}$ can be embedded in $A \rtimes_{\lambda} \mathbf{Z}$.

Proof. Let us take $F=[0,1) \times \mathbf{T}$ as a fundamental domain for $\sigma$, and $H$ as in Lemma 2.1. If $\Phi \in C_{\mu \nu}^{c}$ and $p \in \mathbf{Z}$, then $f_{\Phi, p}(x, y)=\Phi\left(x^{\prime}, y, p\right)$, where $x^{\prime} \in[0,1)$ and $\exp \left(2 \pi i x^{\prime}\right)=\exp (2 \pi i x)$. Therefore $f_{\Phi, p}$ belongs to the $\lambda$-invariant algebra $A$. Thus Theorem 2.3 applies to $A$.

\section{The RANGe OF TRACES ON $K_{0}\left(D_{\mu \nu}^{c}\right)$}

In this section we discuss the range of traces on $K_{0}\left(D_{\mu \nu}^{c}\right)$. We first give a description of tracial states on the algebra $D_{\mu \nu}^{c}$. The techniques involved are an adaptation of those usually employed (see [TO, 3.3]) to relate $\lambda$-invariant probability measures on a $G$-space $X$ to tracial states on $C_{0}(X) \rtimes_{\lambda} G$. Then, by embedding $D_{\mu \nu}^{c}$ in a crossed product as in Section 2, we show that any tracial state $\tau$ on $D_{\mu \nu}^{c}$ induces the same homomorphism on $K_{0}\left(D_{\mu \nu}^{c}\right)$, and that $\tau_{*}\left(K_{0}\left(D_{\mu \nu}^{c}\right)\right)=\mathbf{Z}+2 \mu \mathbf{Z}+2 \nu \mathbf{Z}$.

Lemma 3.1. For each $p \in \mathbf{Z}$ there exist $\Delta_{1}^{p}, \Delta_{2}^{p} \in C_{\mu \nu}^{c}$ such that $\Delta_{i}^{p}(x, y, n)=$ 0 if $n \neq p$, and

i) $\left(\Delta_{1}^{p}\right) * \Delta_{1}^{p}+\left(\Delta_{2}^{p}\right)^{*} * \Delta_{2}^{p}=1=\Delta_{1}^{p} *\left(\Delta_{1}^{p}\right)^{*}+\Delta_{2}^{p} *\left(\Delta_{2}^{p}\right)^{*}$,

ii) $\Delta_{1}^{p} * f *\left(\Delta_{1}^{p}\right)^{*}+\Delta_{2}^{p} * f *\left(\Delta_{2}^{p}\right)^{*}=\lambda_{p}(f)$ for all $f \in C\left(\mathbf{T}^{2}\right)$.

Proof. Let $d \in C(\mathbf{T})$ be such that $0 \leq d \leq 1, d(0)=0$, and $d(1 / 2)=1$. For $p \in \mathbf{Z}$ let $\Delta_{1}^{p}(x, y, n)=d^{1 / 2}(x) \delta_{p}(n)$, for $x \in[0,1], y \in \mathbf{T}$,

$$
\Delta_{2}^{p}(x, y, n)= \begin{cases}(1-d(x))^{1 / 2} \delta_{p}(n) & \forall x \in[0,1 / 2], y \in \mathbf{T}, \\ (1-d(x))^{1 / 2} \exp (-2 \pi i c p(y-p \nu)) \delta_{p}(n) & \forall x \in[1 / 2,1], y \in \mathbf{T},\end{cases}
$$

and extend $\Delta_{i}^{p}$, for $i=1,2$, to continuous functions on $\mathbf{R} \times \mathbf{T} \times \mathbf{Z}$ by setting $\Delta_{i}^{p}(x+1, y, n)=\exp (-2 \pi i c p(y-p \nu)) \Delta_{i}^{p}(x, y) \delta_{p}(n)$, for all $(x, y) \in \mathbf{R} \times \mathbf{T}$. Then

$$
\left[\left(\Delta_{i}^{p}\right)^{*} * \Delta_{i}^{p}\right](x, y, n)=\left|\Delta_{i}^{p}(x+2 p \mu, y+2 p \nu, p)\right|^{2} \delta_{0}(n),
$$

so $\left(\Delta_{1}^{p}\right)^{*} * \Delta_{1}^{p}+\left(\Delta_{2}^{p}\right)^{*} * \Delta_{2}^{p}=\left(\left|\Delta_{1}^{p}\right|^{2}+\left|\Delta_{2}^{p}\right|^{2}\right) \delta_{0}=1$.

Moreover, if $f \in C\left(\mathbf{T}^{2}\right)$, then

$$
\left[\Delta_{i}^{p} * f *\left(\Delta_{i}^{p}\right)^{*}\right](x, y, n)=\left|\Delta_{i}^{p}(x, y, p)\right|^{2} f(x-2 p \mu, y-2 p \nu) \delta_{0}(n),
$$


SO

$$
\Delta_{1}^{p} * f *\left(\Delta_{1}^{p}\right) *+\Delta_{2}^{p} * f *\left(\Delta_{2}^{p}\right)^{*}=\left(\left|\Delta_{1}^{p}\right|^{2}+\left|\Delta_{2}^{p}\right|^{2}\right) \lambda_{p}(f)=\lambda_{p}(f) .
$$

The second equality in i) now follows from taking $f=1$ in ii).

Notation 3.2. Throughout this section $e(a)$ denotes $\exp (2 \pi i a)$, for a real number $a$.

Remark 3.3. It was shown in [AE, 2] that the $\mathrm{C}^{*}$-algebra $D_{\mu \nu}^{c}$ is the crossed product, in the sense of AEE, of $C\left(\mathbf{T}^{2}\right)$ by the Hilbert $\mathrm{C}^{*}$-bimodule $M_{\mu \nu}^{c}$, where $M_{\mu \nu}^{c}=\{f \in C(\mathbf{R} \times \mathbf{T}): f(x+1, y)=e(-c y) f(x, y)\}$ with the structure defined by

$$
\begin{gathered}
(f \cdot \Phi)(x, y)=f(x, y) \Phi(x-2 \mu, y-2 \nu), \quad(\Phi \cdot f)(x, y)=\Phi(x, y) f(x, y), \\
\langle f, g\rangle_{R}(x, y)=\bar{f}(x+2 \mu, y+2 \nu) g(x+2 \mu, y+2 \nu), \\
\langle f, g\rangle_{L}(x, y)=f(x, y) \bar{g}(x, y),
\end{gathered}
$$

for $\Phi \in C\left(\mathbf{T}^{2}\right)$ and $f, g \in M_{\mu \nu}^{c}$.

Since the Hilbert $\mathrm{C}^{*}$-bimodules $M_{\mu \nu}^{c}, M_{\mu+\frac{1}{2}, \nu}^{c}$, and $M_{\mu, \nu+\frac{1}{2}}^{c}$ are clearly isomorphic, it follows that so are the $\mathrm{C}^{*}$-algebras $D_{\mu \nu}^{c}, D_{\mu+\frac{1}{2}, \nu}^{c}$, and $D_{\mu, \nu+\frac{1}{2}}^{c}$.

In $\mathrm{AE}$ the Picard group of $C\left(\mathbf{T}^{2}\right)$ was shown to be the semidirect product of $\operatorname{Aut}\left(C\left(\mathbf{T}^{2}\right)\right)$ by $\left\{M_{00}^{c}: c \in \mathbf{Z}\right\} \cong \mathbf{Z}$. By using this description, it was proved ([AE, $2.2])$ that $D_{\mu \nu}^{c}$ and $D_{\mu^{\prime} \nu^{\prime}}^{c}$ are isomorphic if $(\mu, \nu)$ and $\left(\mu^{\prime}, \nu^{\prime}\right)$ belong to the same orbit under the usual action of $G L_{2}(\mathbf{Z})$ on $\mathbf{T}^{2}$. This result carries over to the case when $(2 \mu, 2 \nu)$ and $\left(2 \mu^{\prime}, 2 \nu^{\prime}\right)$ belong to the same orbit because then, if $A \in G L_{2}(\mathbf{Z})$ is such that $A\left(\begin{array}{c}2 \mu \\ 2 \nu\end{array}\right)=\left(\begin{array}{c}2 \mu^{\prime} \\ 2 \nu^{\prime}\end{array}\right)+\left(\begin{array}{l}k \\ l\end{array}\right)$, for some $k, l \in \mathbf{Z}$, then

$$
A\left(\begin{array}{c}
\mu \\
\nu
\end{array}\right)=\left(\begin{array}{c}
\mu^{\prime}+k / 2 \\
\nu^{\prime}+l / 2
\end{array}\right)
$$

so

$$
D_{\mu \nu}^{c} \cong D_{\mu^{\prime}+\frac{k}{2}, \nu^{\prime}+\frac{l}{2}}^{c} \cong D_{\mu^{\prime}, \nu^{\prime}}^{c}
$$

Lemma 3.4. Let $a, b, p, q$ be non-zero integers such that $\operatorname{gcd}(a, p)=\operatorname{gcd}(b, q)=1$, and let $m=\operatorname{lcm}(p, q)$. Then $\left(\frac{a}{p}, \frac{b}{q}\right)$ and $\left(\frac{1}{m}, 0\right)$ are in the same orbit under the action of $G L_{2}(\mathbf{Z})$ on $\mathbf{T}^{2}$, so $D_{\frac{a}{p}, \frac{b}{q}}^{c} \cong D_{\frac{1}{m}, 0}^{c}$. If $\operatorname{gcd}(a, p)=1$, then $\left(\frac{a}{p}, 0\right),\left(\frac{1}{p}, 0\right)$, and $\left(0, \frac{a}{p}\right)$ belong to the same orbit under the action of $G L_{2}(\mathbf{Z})$, and $D_{\frac{a}{p}, 0}^{c} \cong D_{\frac{1}{p}, 0}^{c} \cong D_{0, \frac{a}{p}}^{c}$.

Proof. Let us write $m=p p^{\prime}=q q^{\prime}$, so $\operatorname{gcd}\left(p^{\prime}, q^{\prime}\right)=1$ and $\operatorname{gcd}\left(a p^{\prime}, b q^{\prime}, m\right)=1$. Then it suffices to show that, if $\operatorname{gcd}(a, b, p)=1$, then $A\left(\frac{a}{p}, \frac{b}{p}\right)=\left(\frac{1}{p}, 0\right)$ for some $A \in$ $G L_{2}(\mathbf{Z})$, viewing $\left(\frac{a}{p}, \frac{b}{p}\right)$ and $\left(\frac{1}{p}, 0\right)$ as elements of $\mathbf{T}^{2}$. This will also show our second statement, since, in $\mathbf{T}^{2},\left(\frac{a}{p}, 0\right)=\left(\frac{a}{p}, \frac{p}{p}\right)$ and $\left(0, \frac{a}{p}\right)=\left(\frac{p}{p}, \frac{a}{p}\right)$. The isomorphisms between the corresponding quantum Heisenberg manifolds will then follow from [AE 2.2].

For $a, b, p$ as above, let $d=\operatorname{gcd}(a, b), \operatorname{sog} \operatorname{gcd}(d, p)=1$. Write $a=a^{\prime} d, b=b^{\prime} d$, and choose integers $r, s$ such that $a^{\prime} r+b^{\prime} s=1$. Then

$$
\left(\begin{array}{cc}
r & s \\
-b^{\prime} & a^{\prime}
\end{array}\right) \in G L_{2}(\mathbf{Z}) \quad \text { and } \quad\left(\begin{array}{cc}
r & s \\
-b^{\prime} & a^{\prime}
\end{array}\right)\left(\begin{array}{c}
\frac{a}{p} \\
\frac{b}{p}
\end{array}\right)=\left(\begin{array}{c}
\frac{d}{p} \\
0
\end{array}\right) .
$$


Now, as elements of $\mathbf{T}^{2},\left(\frac{d}{p}, 0\right)=\left(\frac{d}{p}, \frac{p}{p}\right)$, and $\operatorname{gcd}(d, p)=1$, so, by making use of the result we have just proved, we get that $\left(\frac{d}{p}, 0\right)$ and $\left(\frac{1}{p}, 0\right)$ belong to the same orbit under the action of $G L_{2}(\mathbf{Z})$ on $\mathbf{T}^{2}$.

Remark 3.5. Let $a, m, b, n \in \mathbf{Z}$ be such that $m, n \neq 0, \operatorname{gcd}(a, m)=\operatorname{gcd}(b, n)=1$. Set $p=\frac{1}{2} \operatorname{lcm}(m, n)$ if either $m$ or $n$ is even, and $p=\operatorname{lcm}(m, n)$ otherwise. Then $\left(\frac{2 a}{m}, \frac{2 b}{n}\right)$ and $\left(\frac{1}{p}, 0\right)$ are in the same orbit under the action of $G L_{2}(\mathbf{Z})$ on $\mathbf{T}^{2}$, so $D_{\frac{a}{m}, \frac{b}{n}}^{c}$ is isomorphic to $D_{\frac{1}{2 p}, 0}^{c}$.

Proof. The statement follows from Remark 3.3 and Lemma 3.4.

Notation 3.6. For the remainder of this section, given a quantum Heisenberg manifold $D_{\mu \nu}^{c}$, if both $\mu$ and $\nu$ are rational we assume that $\mu=1 / 2 p$, for $p \in \mathbf{Z}, p>0$, and that $\nu=0$, as in Remark 3.5 If either $\mu$ or $\nu$ is irrational, we set $p=0$.

Let $B_{p}^{c}$ be the $C^{*}$-subalgebra of $D_{\mu \nu}^{c}$ generated by $\left\{\phi \in C_{\mu \nu}^{c}: \operatorname{supp}_{\mathbf{Z}} \phi \subset p \mathbf{Z}\right\}$, and denote by $E_{p}^{c}: D_{\mu \nu}^{c} \longrightarrow B_{p}^{c}$ the conditional expectation on $B_{p}^{c}$ given by

$$
\left(E_{p}^{c} \phi\right)(x, y, n)= \begin{cases}\phi(x, y, n) & \text { if } n \in p \mathbf{Z}, \\ 0 & \text { otherwise }\end{cases}
$$

for $\phi \in C_{\mu \nu}^{c}$.

Proposition 3.7. If $\tau$ is a tracial state on $D_{\mu \nu}^{c}$, then $\tau=\tau \circ E_{p}^{c}$.

Proof. We show that $\tau\left(\Phi \delta_{n}\right)=0$, for $n \notin p \mathbf{Z}$. Since for $\Delta_{i}^{n}$ as in Lemma 3.1 we have that

$$
\phi \delta_{n}=\phi \delta_{n} *\left(\Delta_{1}^{n}\right)^{*} * \Delta_{1}^{n}+\phi \delta_{n} *\left(\Delta_{2}^{n}\right)^{*} * \Delta_{2}^{n},
$$

and $\phi \delta_{n} *\left(\Delta_{i}^{n}\right)^{*} \in C\left(\mathbf{T}^{2}\right)$, for $i=1,2$, it suffices to show that $\tau\left(g * \Delta_{i}^{n}\right)=0$, for all $g \in C\left(\mathbf{T}^{2}\right), i=1,2$, and $n \notin p \mathbf{Z}$. For a fixed $n \notin p \mathbf{Z}$, we can assume that $g=f^{2}$ for some positive function $f$ satisfying $\operatorname{supp}(f) \cap \operatorname{supp}\left(\lambda_{n} f\right)=\emptyset$, because, since in this case $\lambda^{n}(x, y) \neq(x, y)$ for all $(x, y) \in \mathbf{T}^{2}$, any function $g \in C\left(\mathbf{T}^{2}\right)$ is the linear combination of functions satisfying those conditions. So let $g \in C\left(\mathbf{T}^{2}\right)$ be as above. Then

$$
\tau\left(g * \Delta_{i}^{n}\right)=\tau\left(f^{2} * \Delta_{i}^{n}\right)=\tau\left(f * f * \Delta_{i}^{n}\right)=\tau\left(f * \Delta_{i}^{n} * f\right)=0,
$$

because

$$
f * \Delta_{i}^{n} * f=f \Delta_{i}^{n}\left(\lambda_{n} f\right)=0 .
$$

This shows that $\tau=\tau \circ E_{p}^{c}$, since both sides are continuous and agree on $C_{\mu \nu}^{c}$.

Proposition 3.8. Let $D_{\mu \nu}^{c}, p, B_{p}^{c}$, and $E_{p}^{c}$ be as in Notation[3.6] and let $\gamma: B_{p}^{c} \longrightarrow$ $B_{p}^{c}$ be given by

$$
\gamma \phi=\Delta_{1}^{1} * \phi *\left(\Delta_{1}^{1}\right)^{*}+\Delta_{2}^{1} * \phi *\left(\Delta_{2}^{1}\right)^{*},
$$

for $\phi \in B_{p}^{c}$ and $\Delta_{i}^{1}, i=1,2$, as in Lemma 3.1 .

Then, for $\phi \in B_{p}^{c}$ compactly supported on $\mathbf{Z}$,

$$
(\gamma \phi)(x, y, m)= \begin{cases}\phi(x-2 \mu, y-2 \nu, 0) \delta_{0}(m) & \text { if } p=0, \\ e(-c n y) \phi(x-1 / p, y, n p) \delta_{n p}(m) & \text { if } p \neq 0 .\end{cases}
$$

Also, the correspondence $\tau \mapsto \tau \circ E_{p}^{c}$ is a bijection between the set of $\gamma$-invariant tracial states on $B_{p}^{c}$ and tracial states on $D_{\mu \nu}^{c}$. 
Proof. If $\tau$ is a trace on $D_{\mu \nu}^{c}$ then, by Proposition 3.7 we have that $\tau=\tau \circ E_{p}^{c}$, and the restriction of $\tau$ to $B_{p}^{c}$ is $\gamma$-invariant because

$$
\tau(\gamma \phi)=\tau\left[\left(\Delta_{1}^{1}\right)^{*} * \Delta_{1}^{1} * \phi+\left(\Delta_{2}^{1}\right)^{*} * \Delta_{2}^{1} * \phi\right]=\tau(\phi) .
$$

Now, for $\phi \in B_{p}^{c}$ compactly supported on $\mathbf{Z}$, we have

$$
\begin{aligned}
& {\left[\Delta_{i}^{1} * \phi *\left(\Delta_{i}^{1}\right)^{*}\right](x, y, n p)} \\
& \quad=\Delta_{i}^{1}(x, y, 1) \phi(x-2 \mu, y-2 \nu, n p) \overline{\Delta_{i}^{1}(x-2 n p \mu, y-2 n p \nu, 1)}
\end{aligned}
$$

so

$$
(\gamma \phi)(x, y, m)= \begin{cases}\phi(x-2 \mu, y-2 \nu, 0) \delta_{0}(m) & \text { if } p=0, \\ e(-c n y) \phi(x-1 / p, y, n p) \delta_{n p}(m) & \text { if } p \neq 0 .\end{cases}
$$

Now let $\tau$ be a $\gamma$-invariant tracial state on $B_{p}^{c}$. Since $\tau \circ E_{p}^{c}$ is a state, we only need to show that $\tau \circ E_{p}^{c}(\phi * \psi)=\tau \circ E_{p}^{c}(\psi * \phi)$, for $\phi=f \delta_{k}, \psi=g \delta_{l}$. We can assume that $k+l \in p \mathbf{Z}$, since otherwise $E_{p}^{c}(\phi * \psi)=0=E_{p}^{c}(\psi * \phi)$.

If $p \neq 0$, we take $\phi$ and $\psi$ as above, with $k+l=n p$, and we have

$$
\begin{aligned}
{\left[\gamma^{-k}(\phi * \psi)\right](x, y, m) } & =e(c n k y) f(x+k / p, y) g(x, y) \delta_{n p}(m) \\
& =g(x, y) f(x+(k-n p) / p, y) \delta_{n p}(m) \\
& =g(x, y) f(x-l / p, y) \delta_{n p}(m) \\
& =(\psi * \phi)(x, y, m) .
\end{aligned}
$$

So $\left(\tau \circ E_{p}^{c}\right)(\phi * \psi)=\tau(\phi * \psi)=\tau\left(\gamma^{k}(\psi * \phi)\right)=\tau(\psi * \phi)=\left(\tau \circ E_{p}^{c}\right)(\psi * \phi)$. Similar computations prove the case $p=0$.

Proposition 3.9. Given a quantum Heisenberg manifold $D_{\mu \nu}^{c}$, let $p, B_{p}^{c}$ and $E_{p}^{c}$ be as in Remark 3.6. Then $B_{p}^{c} \cong C\left(\mathbf{T}^{2}\right)$ if $p=0$, and $B_{p}^{c} \cong D_{0,0}^{c p}$ if $p \neq 0$.

Proof. It is clear that $B_{p}^{c} \cong C\left(\mathbf{T}^{2}\right)$ for $p=0$. If $p \neq 0$, set $J: B_{p}^{c} \longrightarrow D_{0,0}^{c p}$,

$$
J \phi(x, y, n)=u_{p}(n, y) \phi(x, y, n p),
$$

for $\phi \in B_{p}^{c} \cap C_{\frac{1}{2 p}, 0}^{c}$, where $u_{p}(n, y)=e\left(-\frac{1}{2} \operatorname{cpn}(n-1) y\right)$.

Notice that

$$
(J \phi)(x+1, y, n)=u_{p}(n, y) e(-c n p y) \phi(x, y, n p)=e(-c p n y)(J \phi)(x, y, n),
$$

so $J \phi \in D_{0,0}^{c p}$, for $\phi \in B_{p}^{c} \cap C_{\frac{1}{2 p}, 0}^{c}$.

Let $\Pi$ and $\sigma$ denote, respectively, the faithful representations ([RF3] ) of $D_{\frac{1}{2 p}, 0}^{c}$ and $D_{0,0}^{c p}$ on $L^{2}(\mathbf{R} \times \mathbf{T} \times \mathbf{Z})$ given by

$$
\begin{gathered}
\left(\Pi_{\phi} \xi\right)(x, y, n)=\sum_{q} \phi(x+n / p, y, q p) \xi(x, y, n-q p), \\
\left(\sigma_{\psi} \eta\right)(x, y, n)=\sum_{q} \phi(x, y, q) \eta(x, y, n-q),
\end{gathered}
$$

for $\phi \in C_{1 / p, 0}^{c}, \psi \in C_{0,0}^{c p}, \xi, \eta \in L^{2}(\mathbf{R} \times \mathbf{T} \times \mathbf{Z})$.

Let $U: L^{2}(\mathbf{R} \times \mathbf{T} \times \mathbf{Z}) \longrightarrow \bigoplus_{0}^{p-1} L^{2}(\mathbf{R} \times \mathbf{T} \times \mathbf{Z})$ be given by

$$
(U \xi)_{i}(x, y, n)=\overline{u_{p}(-n, y)} \xi(x, y, n p+i),
$$


for $\xi \in L^{2}(\mathbf{R} \times \mathbf{T} \times \mathbf{Z})$. It is easily checked that $U$ is unitary and that

$$
\left[U^{*}\left(\left(\eta_{i}\right)\right)\right](x, y, n)=u_{p}(-k, y) \eta_{i}(x, y, k) \quad \text { for } n=k p+i, 0 \leq i<p .
$$

Now,

$$
\begin{aligned}
& {\left[U \Pi_{\phi} U^{*}\left(\left(\eta_{i}\right)\right)\right]_{j}(x, y, n) } \\
&=\overline{u_{p}(-n, y)}\left(\Pi_{\phi} U^{*}\left(\left(\eta_{i}\right)\right)\right)(x, y, n p+j) \\
&=\sum_{q} \overline{u_{p}(-n, y)} \phi(x+(n p+j) / p, y, q p)\left(U^{*}\left(\left(\eta_{i}\right)\right)(x, y,(n-q) p+j)\right. \\
&=\sum_{q} \overline{u_{p}(-n, y)} e(-c n p q y) \phi(x+j / p, y, q p) \eta_{j}(x, y, n-q) u_{p}(q-n, y) \\
&=\sum_{q} u_{p}(q, y) \phi(x+j / p, y, q p) \eta_{j}(x, y, n-q) \\
&=\sum_{q}(J \phi)(x+j / p, y, q) \eta_{j}(x, y, n-q) \\
&=\left[\sigma_{\left(\delta^{j}(J \phi)\right)}\left(\eta_{j}\right)\right](x, y, n-q),
\end{aligned}
$$

where $\left(\delta^{j} \psi\right)(x, y, n)=\psi(x+j / p, y, n)$ for all $\psi \in C_{0,0}^{c p}$, and $0 \leq j<p$. Notice that $\delta^{j}$ defines an automorphism of $D_{0,0}^{c p}$ : apply AB2 1.1] to define $\delta^{j}$ on $C_{b}(\mathbf{R} \times \mathbf{T}) \rtimes_{i d} \mathbf{Z}$ and then check that $D_{0,0}^{c p}$ is invariant under it. Thus $U$ intertwines $\Pi_{\phi}$ and $\bigoplus_{j}\left(\sigma \circ \delta^{j}\right)(J \phi)$, which shows that $J$ extends to an isomorphism.

Remark 3.10. Recall $([\overline{\mathrm{RF}}])$ that, for a positive integer $c$, the $\mathrm{C}^{*}$-algebra $D_{0,0}^{c}$ is isomorphic to the (commutative) Heisenberg manifold $C\left(M^{c}\right)$, where $M^{c}$ is the quotient space of $\mathbf{R} \times \mathbf{T}^{2}$ under the equivalence relation given by

$$
(x, y, z) \cong\left(x^{\prime}, y^{\prime}, z^{\prime}\right) \text { if and only if }\left(x^{\prime}, y^{\prime}, z^{\prime}\right)=(x+k, y, z+c k y)
$$

for some $k \in \mathbf{Z}$, and $(x, y, z),\left(x^{\prime}, y^{\prime}, z^{\prime}\right) \in \mathbf{R} \times \mathbf{T}^{2}$ (viewing $\mathbf{T}$ as $\mathbf{R} / \mathbf{Z}$ ).

The isomorphism is obtained by taking Fourier transform in the third variable, that is, $F: C\left(M^{c}\right) \longrightarrow D_{0,0}^{c},(F f)(x, y, n)=\int_{\mathbf{T}} e(-n z) f(x, y, z) d z$.

Corollary 3.11. Given a quantum Heisenberg manifold $D_{\mu \nu}^{c}$, let $p, B_{p}^{c}$, and $E_{p}^{c}$ be as in Notation 3.6 . There is a bijective correspondence between tracial states on $D_{\mu \nu}^{c}$ and $\gamma$-invariant probability measures on $X$, where

$$
X=\mathbf{T}^{2}, \quad \gamma(x, y)=(x+2 \mu, y+2 \nu),
$$

if either $\mu$ or $\nu$ is irrational, and

$$
X=M^{c p}, \quad \gamma(x, y, z)=(x+1 / p, y, z+c y)
$$

if $\mu=\frac{1}{2 p}, \nu=0$.

The correspondence is given by $m \mapsto \tau_{m} \circ E_{p}^{c}$, where $\tau_{m}(f)=\int_{X} f d m$, once $B_{p}^{c}$ is identified with $C(X)$, according to Proposition 3.9 and Remark 3.10

Proof. It is easily checked that the formula above is the formula for $\gamma$ in Proposition 3.8, when one keeps track of the isomorphisms $J$ and $F$ in Proposition 3.9 and Remark 3.10 respectively.

Corollary 3.12. If $\{1, \mu, \nu\}$ is linearly independent over the field of rational numbers, then the trace corresponding to Haar measure on $\mathbf{T}^{2}$ is the only tracial state on $D_{\mu \nu}^{c}$. 
Proof. Under the conditions above, $\mu$ and $\nu$ are irrational, and the $\lambda$-orbits in $\mathbf{T}^{2}$ are dense. Therefore Haar measure is the only $\lambda$-invariant measure on $\mathbf{T}^{2}$. The uniqueness of the trace now follows from Corollary 3.11 .

Remark 3.13. For $D_{\mu \nu}^{c}$ and $p$ as in Notation 3.6, we can identify $C\left(\mathbf{T}^{2}\right)$ with the $\mathrm{C}^{*}$-algebra consisting of the $\delta_{0}$-maps in $B_{p}^{c}$. It follows from Proposition 3.8 that, for any value of $p$, a trace on $D_{\mu \nu}^{c}$ induces a probability measure $m_{\tau}$ on $\mathbf{T}^{2}$, invariant under translation by $(2 \mu, 2 \nu)$, and such that $\tau(f)=\int_{\mathbf{T}^{2}} f d m_{\tau}$, for all $f \in C\left(\mathbf{T}^{2}\right)$.

Proposition 3.14. Let $D_{\mu \nu}^{c}$ be a quantum Heisenberg manifold, where $(\mu, \nu)=$ $\left(\frac{1}{2 p}, 0\right)$ as in Remark 3.5 if $\mu$ and $\nu$ are rational. Then, in the notation of Corollary 2.4, all traces on $D_{\mu \nu}^{c}$ arise from restricting traces on $A \rtimes_{\lambda} \mathbf{Z}$, where $D_{\mu \nu}^{c}$ is embedded in $A \rtimes_{\lambda} \mathbf{Z}$ as in Theorem 2.3.

Proof. Let $A$ be as in Corollary 2.4 Notice that the embedding $J$ in Theorem 2.3 maps the $\mathrm{C}^{*}$-algebra $B_{p}^{c}$ defined in Notation 3.6 to the commutative $\mathrm{C}^{*}$-subalgebra $B$ of $A \rtimes_{\lambda} \mathbf{Z}$ generated by $\left\{\phi \in C_{c}(\mathbf{Z}, A): \operatorname{supp}_{\mathbf{Z}} \phi \subset p \mathbf{Z}\right\}$, and that $J$ is the identity when restricted to $C\left(\mathbf{T}^{2}\right) \subset B_{p}^{c}$ as in Corollary[3.13 So, if either $\mu$ or $\nu$ is irrational, then the statement follows from Proposition [3.7, Corollary [3.11, and [TO, 3.3.9].

If $(\mu, \nu)=\left(\frac{1}{2 p}, 0\right)$, given a trace $\tau$ on $D_{\frac{1}{2 p}, 0}^{c}$, let $S$ denote the set of states on $B$ extending $\tau_{0} \circ J^{-1}$ on $J\left(B_{p}^{c}\right)$, where $\tau_{0}$ denotes the restriction of $\tau$ to $B_{p}^{c}$.

Let $T: B \longrightarrow B$ be given by

$$
T(a)=J\left(\Delta_{1}^{1}\right) * a * J\left(\Delta_{1}^{1}\right)^{*}+J\left(\Delta_{2}^{1}\right) * a * J\left(\Delta_{2}^{1}\right)^{*},
$$

with $\Delta_{i}^{1}, i=1,2$, as in Lemma 3.1 , and $J$ as in Theorem 2.3, and set $T^{*}: B^{*} \longrightarrow$ $B^{*}, T^{*}(\rho)=\rho \circ T$. If $\rho \in S$, then $T^{*}(\rho)$ is positive and $\left\|T^{*}(\rho)\right\|=\left[T^{*}(\rho)\right](1)=$ $\rho(1)=1$, by Lemma 3.1 Besides, the restriction of $T^{*}(\rho)$ to $J\left(B_{p}^{c}\right)$ is $\tau_{0}$ by Proposition 3.8. Then $T^{*}(S) \subset S$, and $S$ is a $w^{*}$-compact, convex, non-empty set, so it follows from Markov's fixed-point theorem that there exists $\tau_{1} \in S$ such that $T^{*}\left(\tau_{1}\right)=\tau_{1}$.

We next show that if $P$ denotes the conditional expectation $P: A \rtimes_{\lambda} \mathbf{Z} \longrightarrow B$ given by

$$
(P \phi)(x, y, n)= \begin{cases}\phi(x, y, n) & \text { if } n \in p \mathbf{Z} \\ 0 & \text { otherwise }\end{cases}
$$

for $\phi \in C_{c}(\mathbf{Z}, A)$, then $\tau_{1} \circ P$ is a trace on $A \rtimes_{\lambda} \mathbf{Z}$. This will end the proof, because the diagram

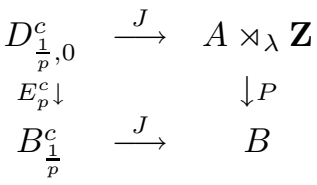

commutes, and, by Proposition $3.7 \tau_{0}=\tau \circ E_{p}^{c}$, so

$$
\tau=\tau_{0} \circ E_{p}^{c}=\tau_{1} \circ J \circ E_{p}^{c}=\tau_{1} \circ P \circ J .
$$

Now, $\tau_{1}$ is a state, so it suffices to show that $(\tau \circ P)(\phi * \psi)=(\tau \circ P)(\psi * \phi)$, for $\phi=F \delta_{k}, \psi=G \delta_{l}$, for some $F, G \in A$. 
First notice that if $H \in A$, and $n \in \mathbf{Z}$, then $T\left(H \delta_{n p}\right)=(\lambda H) \delta_{n p}$. In fact,

$$
\begin{aligned}
T( & \left.H \delta_{n p}\right)(x, y, m) \\
& =\sum_{i=1}^{2}\left[J\left(\Delta_{i}^{1}\right) * H \delta_{n p} * J\left(\Delta_{i}^{1}\right)^{*}\right](x, y, m) \\
& =\sum_{i=1}^{2} J\left(\Delta_{i}^{1}\right)(x, y, 1) H\left(x-\frac{1}{p}, y, n p\right) J\left(\Delta_{i}^{1}\right)^{*}\left(x-\frac{1}{p}-n, y,-1\right) \delta_{n p}(m) \\
& =\sum_{i=1}^{2}\left|J\left(\Delta_{i}^{1}\right)(x, y, 1)\right|^{2}(\lambda H)(x, y) \delta_{n p}(m) \\
& =\sum_{i=1}^{2}\left|\left(\Delta_{i}^{1}\right)(x, y, 1)\right|^{2}(\lambda H)(x, y) \delta_{n p}(m) \\
& =\left[(\lambda H) \delta_{n p}\right](x, y, m) .
\end{aligned}
$$

Now, for $\phi$ and $\psi$ as above, we can assume that $k+l=n p$ for some $n \in \mathbf{Z}$, since otherwise $P(\phi * \psi)=0=P(\psi * \phi)$. In this case

$$
\begin{aligned}
{\left[T^{k}(\psi * \phi)\right](x, y, m) } & =(\psi * \phi)\left(x-\frac{k}{p}, y, m\right) \\
& =G\left(x-\frac{k}{p}, y\right) F\left(x-\frac{l}{p}-\frac{k}{p}, y\right) \delta_{n p}(m) \\
& =F(x, y) G\left(x-\frac{k}{p}, y\right) \delta_{n p}(m) \\
& =(\phi * \psi)(x, y, m) .
\end{aligned}
$$

Therefore

$$
\left(\tau_{1} \circ P\right)(\phi * \psi)=\tau_{1}(\phi * \psi)=\tau_{1}\left[T^{k}(\psi * \phi)\right]=\tau_{1}(\psi * \phi)=\tau_{1} \circ P(\psi * \phi),
$$

as we wanted to show.

Lemma 3.15. If $\mu \leq 1 / 2$ and $m$ is a $\lambda$-invariant probability measure on $\mathbf{T}^{2}$, then $m([0,2 \mu) \times \mathbf{T})=2 \mu$.

Proof. First notice that the analogous result holds for T. Fix a real number $\alpha \in$ $[0,1]$. If $v$ is a measure on $\mathbf{T}$ invariant under translation by $\alpha$, then $v([0, \alpha))=\alpha$. If $\alpha$ is irrational, then $v$ is Haar measure on $\mathbf{T}$, and the result is obviously true. If $\alpha$ is rational, $\alpha=p / q$, for $p, q \in \mathbf{Z}$, with $(p, q)=1$, then $\mathbf{T}$ is the disjoint union of the intervals $I_{i}=[i / q,(i+1) / q), i=0,1, \ldots, q-1$.

Now, for all $i, I_{i}$ can be obtained by translating $I_{0}$ by $\alpha$ an appropriate number of times . Therefore $v\left(I_{i}\right)=v\left(I_{0}\right)=1 / q$, for all $i=1, \ldots, q-1$, and it follows that $v([0, \alpha))=v([0, p / q))=p / q=\alpha$.

Now let $m$ be a $\lambda$-invariant probability measure on $\mathbf{T}^{2}$. Define a probability measure $v$ on $\mathbf{T}$ by setting $v(X)=m(X \times \mathbf{T})$.

Then $v(A+2 \mu)=m((A+2 \mu) \times \mathbf{T})=m(\lambda(A \times \mathbf{T}))=m(A \times \mathbf{T})=v(A)$.

It follows now that $m([0,2 \mu) \times \mathbf{T})=v([0,2 \mu))=2 \mu$.

Theorem 3.16. All tracial states $\tau$ on $D_{\mu \nu}^{c}$ induce the same homomorphism $\tau_{*}$ on $K_{0}\left(D_{\mu \nu}^{c}\right)$. Moreover, $\tau_{*}\left(K_{0}\left(D_{\mu \nu}^{c}\right)\right)=\mathbf{Z}+2 \mu \mathbf{Z}+2 \nu \mathbf{Z}$. 
Proof. For a tracial state $\tau$ on $D_{\mu \nu}^{c}$, we denote by $\tau^{\prime}$ an extension of $\tau$ to $A \rtimes_{\lambda} \mathbf{Z}$, as in Proposition 3.14. We have the following short exact sequence ([PM, 3,4]):

$$
0 \longrightarrow \tau_{*}\left(K_{0}(A)\right) \stackrel{i}{\longrightarrow} \tau_{*}^{\prime}\left(K_{0}\left(A \rtimes_{\lambda} \mathbf{Z}\right)\right) \stackrel{q}{\longrightarrow} \Delta_{\tau}^{\lambda}(K) \longrightarrow 0
$$

where $K=\left\{u \in \mathcal{U}_{1}(A):[u]_{K_{1}} \in \operatorname{ker}\left(1-\lambda_{*}\right\}\right), i$ and $q$ are inclusion and projection on $\mathbf{R} / \tau_{*}\left(K_{0}(A)\right)$ respectively, $\Delta_{\tau}^{\lambda}(u)=q\left[\Delta_{\tau}\left(u \lambda\left(u^{-1}\right)\right)\right]$, and $\Delta_{\tau}:\left(\mathcal{U}_{1}\right)_{0} \longrightarrow R$ is defined by $\Delta_{\tau}\left(e^{2 \pi i y}\right)=\tau(y)$, for $y$ self-adjoint.

Let us relabel the set $X=(2 \mu \mathbf{Z}+\mathbf{Z}) \cap(0,1)$ so that $X=\left\{x_{i}: i \in N\right\}$. Let $A_{n}$ be the smallest $\mathrm{C}^{*}$-subalgebra of $L^{\infty}\left(\mathbf{T}^{2}\right)$ generated by $C\left(\mathbf{T}^{2}\right)$ and $\chi_{\left[0, x_{i}\right] \times \mathbf{T}}$, for $i=1, \ldots, n$. Then $A_{1} \subseteq A_{2} \subseteq \ldots \subseteq A_{n} \subseteq \ldots$, and $A$ is the direct limit of $\left\{A_{n}\right\}$.

Now, $A_{n} \simeq \bigoplus_{j=0}^{j=n} C\left(\left[x_{i_{j}}, x_{i_{j+1}}\right] \times \mathbf{T}\right)$, where $\left\{x_{i_{j}}\right\}_{j=1}^{n}=\left\{x_{i}\right\}_{i=1}^{n}, x_{i_{0}}=0, x_{i_{n+1}}=$ 1 , and $x_{i_{j}}<x_{i_{j+1}}$ for all $j=0,1, \ldots, n$.

Since $[a, b] \times \mathbf{T}$ can be deformed to $\mathbf{T}$, it follows that $K_{j}\left(A_{n}\right)=\mathbf{Z}^{n+1} \forall n \in N$, $j=1,2$. The set

$$
\left\{\left[\chi_{\left[x_{i}, x_{j}\right] \times \mathbf{T}}\right]_{K_{0}}: x_{i}, x_{j} \in X \cup\{0,1\}, x_{i}<x_{j}\right\}
$$

is a generator of $K_{0}(A)$, and any arbitrary element of $K_{1}(A)$ has a representative $u$ of the form

$$
u(x, y)=e\left(n_{i} y\right) \quad \text { if } x \in\left[t_{i}, t_{i+1}\right)
$$

for a partition $0=t_{0}<t_{1}<\ldots<t_{n}=1,\left\{t_{i}\right\}_{i=1}^{i=n-1} \subset X$, and integers $n_{i}, i=$ $0, \ldots, n-1$.

Now, by Lemma 3.15 and Remark 3.13 , we have that $\tau_{*}\left(K_{0}(A)\right) \subseteq \mathbf{Z}+2 \mu \mathbf{Z}$. Since $i d$ and $\chi_{\left[0,2 \mu+k_{0}\right] \times \mathbf{T}} \in A$ for some $k_{0}$, the equality holds, and $\tau_{*}\left(K_{0}(A)\right)=\mathbf{Z}+2 \mu \mathbf{Z}$.

Let us now find the elements $[u]_{K_{1}} \in K_{1}(A)$ that are left fixed by $\lambda_{*}$, where $u$ is as above.

For $[u]_{K_{1}} \in K_{1}(A)$,

$$
\lambda_{k}(u)(x, y)=u(x-2 k \mu, y-2 k \nu),
$$

that is,

$$
\lambda_{k}(u)(x, y)=e\left(n_{i}(y-2 k \nu)\right), \quad \text { where } x-2 k \mu \in\left[x_{i}, x_{i+1}\right) .
$$

Fix $a \in\left[x_{0}, x_{1}\right)$. If $\mu$ is irrational, then for all $i=0,1, \ldots, n$ there exists $k_{i} \in \mathbf{Z}$ such that $a-2 k_{i} \mu \in\left[x_{i}, x_{i+1}\right)$ and $\left(\lambda_{k_{i}}(u)\right)(a, y)=e\left(n_{i}\left(y-2 k_{i} \nu\right)\right)$. It is clear now that $[u]_{K_{1}}=\left[\lambda_{k}(u)\right]_{K_{1}}$ for all $k \in \mathbf{Z}$ if and only if $n_{i}=n_{0}$ for all $i=0,1, \ldots, n$. Therefore $\Delta_{\tau^{\prime}}\left(u \lambda\left(u^{-1}\right)\right)=\tau\left(2 n_{0} \nu . I d\right)=2 n_{0} \nu$, and it follows that $\Delta_{\tau}^{\lambda}(K)=2 \nu \mathbf{Z}$.

If $2 \mu$ is rational, $2 \mu=p / q$, where $p, q \in \mathbf{Z},(p, q)=1$, then $X=\{i / q: i=0, \ldots, q\}$ and $u$ is of the form

$$
u(x, y)=e\left(n_{k} y\right) \quad \text { for } x \in I_{k}=[k / q,(k+1) / q], k=0,1, \ldots, q-1 .
$$

Translation by $p / q$ gives a transitive action of $\mathbf{Z}_{q}$ on the set $\left\{I_{k}\right\}$, since $(p, q)=1$, so the same reasoning as for the irrational case applies, and $[u]_{K_{1}}=[\lambda u]_{K_{1}}$ if and only if $u(x, y)=e(n y)$ for all $x, y$. Then, as above, $\Delta_{\tau}^{\lambda}(K)=2 \nu \mathbf{Z}$.

Therefore the short exact sequence above splits, and $\tau_{*}^{\prime}\left(K_{0}\left(A \rtimes_{\lambda} \mathbf{Z}\right)\right)=\mathbf{Z}+$ $2 \mu \mathbf{Z}+2 \nu \mathbf{Z}$, so $\tau_{*}\left(K_{0}\left(D_{\mu \nu}^{c}\right)\right) \subseteq \mathbf{Z}+2 \mu \mathbf{Z}+2 \nu \mathbf{Z}$.

Now, it is shown in [PM, 2,3,4] that, for $[p] \in K_{0}\left(A \rtimes_{\lambda} \mathbf{Z}\right)$, the choice of $u \in K$ such that $q\left(\tau_{*}^{\prime}([p])\right)=\Delta_{\tau}^{\lambda}(u)$ does not depend on $\tau$, and we just proved that $\Delta_{\tau}^{\lambda}(u)$ does not depend on $\tau$ either.

So we have $\tau_{*}^{\prime}[p]=\Delta_{\tau}^{\lambda}(u)+\tau_{*}\left(\left[p_{0}\right]\right)$, for some $p_{0} \in K_{0}(A)$. We next show that $\tau_{*}\left(\left[p_{0}\right]\right)$ is independent of $\tau$ as well. The preceding remarks show that $\left[p_{0}\right]$ has a 
representative $h \in \bigoplus C\left(\left[x_{i_{j}}, x_{i_{j+1}}\right] \times \mathbf{T}\right)$, so $h$ is constant on $\left[x_{i_{j}}, x_{i_{j+1}}\right] \times \mathbf{T}$ for each $j$. Our claim then follows from Lemma 3.15 since $\tau_{*}\left(\left[p_{0}\right]\right)=\int_{\mathbf{T}^{2}} h d m_{\tau}$. So $\tau_{*}$ does not depend on $\tau$, and $\tau_{*}\left(K_{0}\left(D_{\mu \nu}^{c}\right)\right) \subset \mathbf{Z}+2 \mu \mathbf{Z}+2 \nu \mathbf{Z}$. Finally, the equality holds because it is attained for the trace induced by Haar measure on $\mathbf{T}^{2}$ ([AB1]).

Corollary 3.17. Given a quantum Heisenberg manifold $D_{\mu \nu}^{c}$, let $G_{\mu \nu}$ denote the group $\mathbf{Z}+2 \mu \mathbf{Z}+2 \nu \mathbf{Z}$.

If $G_{\mu \nu}$ has rank 1 or 3, then $D_{\mu \nu}^{c}$ and $D_{\mu^{\prime} \nu^{\prime}}^{c}$ are isomorphic if and only if $(2 \mu, 2 \nu)$ and $\left(2 \mu^{\prime}, 2 \nu^{\prime}\right)$ belong to the same orbit under the usual action of $G L_{2}(\mathbf{Z})$ on $\mathbf{T}^{2}$.

Proof. If $D_{\mu \nu}^{c} \cong D_{\mu^{\prime} \nu^{\prime}}^{c}$, then $G_{\mu \nu}=G_{\mu^{\prime} \nu^{\prime}}$, by Theorem 3.16 If $3=\operatorname{rank}\left(G_{\mu \nu}\right)=$ $\operatorname{rank}\left(G_{\mu^{\prime} \nu^{\prime}}\right)$, then $G_{\mu \nu}=G_{\mu^{\prime} \nu^{\prime}}$ implies (see, for instance, [PA1, 2.13]) that $(2 \mu, 2 \nu)$ and $\left(2 \mu^{\prime}, 2 \nu^{\prime}\right)$ are in the same orbit under the action of $G L_{2}(\mathbf{Z})$.

If $1=\operatorname{rank}\left(G_{\mu \nu}\right)=\operatorname{rank}\left(G_{\mu^{\prime} \nu^{\prime}}\right)$, then $\mu, \nu, \mu^{\prime}$, and $\nu^{\prime}$ are rational numbers. By virtue of Remark 3.5 we can assume that $(\mu, \nu)=\left(\frac{1}{2 p_{1}}, 0\right)$ and $\left(\mu^{\prime}, \nu^{\prime}\right)=\left(\frac{1}{2 p_{2}}, 0\right)$ for some $p_{1}, p_{2} \in \mathbf{Z}, p_{1}, p_{2} \neq 0$. Now the equality

$$
\mathbf{Z}+\frac{1}{p_{1}} \mathbf{Z}=G_{\mu, \nu}=G_{\mu^{\prime}, \nu^{\prime}}=\mathbf{Z}+\frac{1}{p_{2}} \mathbf{Z}
$$

implies that $\frac{1}{p_{1}} \mathbf{Z}=\frac{1}{p_{2}} \mathbf{Z}$, so $p_{1}= \pm p_{2}$, and the result follows.

The converse statement was shown in [AE, Thm. 2.2] (see also Remark 3.3).

\section{REFERENCES}

[AB1] Abadie, B. "Vector bundles" over quantum Heisenberg manifolds. Algebraic Methods in Operator Theory, Birkhäuser, 1994, pp. 307-315. MR 95j:58006

[AB2] Abadie, B. Generalized fixed-point algebras of certain actions on crossed-products. Pacific Journal of Mathematics, Vol 171, No.1, (1995), pp. 1-21. MR 96m:46121

[AEE] Abadie, B.; Eilers, S.; Exel, R. Morita equivalence for crossed products by Hilbert C* bimodules. Transactions of the American Mathematical Society, Vol. 350, No. 8, (1998), pp. 3043-3054. MR 98k:46109

[AE] Abadie, B.; Exel, R. Hilbert $\mathrm{C}^{*}$-bimodules over commutative $\mathrm{C}^{*}$-algebras and an isomorphism condition for quantum Heisenberg manifolds. Reviews in Mathematical Physics, Vol. 9, No 4 (1997) 411-423. MR 98d:46060

[CU] Cuntz, J. K-theory for certain $C^{*}$-algebras II. Journal of Operator Theory, 5 (1981) 101108. MR 84k:46053

[PA1] Packer, J. $C^{*}$-algebras generated by projective representations of the discrete Heisenberg group. Journal of Operator Theory, 18 (1987), 41-66. MR 89h:46079

[PA2] Packer, J. Strong Morita equivalence for Heisenberg $C^{*}$-algebras and the positive cones of their $K_{0}$-groups. Canadian Journal of Mathematics, Vol XL, No. 4 (1988), 833-864. MR 89k:46085

[PD] Pedersen, G. $C^{*}$-algebras and their automorphism groups. Academic Press, London/New York/San Francisco (1979). MR 81e:46037

[PM] Pimsner, M. Ranges of traces on $K_{0}$ of reduced crossed products by free groups. "Operator algebras and their connections with topology and ergodic theory" Lecture Notes in Mathematics, No 1132, Springer (1985), pp. 374-408. MR 87j:46131

[PV] Pimsner, M. ; Voiculescu, D. Embedding the irrational rotation $C^{*}$-algebra into an AF algebra. Journal of Operator Theory, 4 (1980), 201-210. MR 82d:46086]

[RF1] Rieffel, M. $C^{*}$-algebras associated with irrational rotations. Pacific Journal of Mathematics Vol. 93, 2, (1981), 415-429. MR 83b:46087

[RF2] Rieffel, M. The cancellation theorem for projective modules over irrational rotation $C^{*}$ algebras. Proc. London Math. Soc. (3) 47 (1983), 285-302. MR 85g:46085

[RF3] Rieffel, M. Deformation quantization of Heisenberg manifolds. Commun. Math. Phys. 122 (1989), 531-562. MR 90e:46060 
[RF4] Rieffel, M. Proper actions of groups on $C^{*}$-algebras. Mappings of operator algebras, Proc. Japan-US joint seminar, Birkhäuser (1990), pp. 141-182. MR 92i:46079]

[TO] Tomiyama, J. Invitation to $C^{*}$-algebras and topological dynamics. World Scientific Advanced Series in Dynamical Systems, Vol. 3, World Sci. Publ., Singapore, 1987. MR 90a:46177

Centro de Matemáticas, Facultad de Ciencias, Iguá 4225, CP 11 400, Montevideo, URUGUAY

E-mail address: abadie@cmat.edu.uy 